\title{
High resolution quantitative multi-species hydrocarbon gas sensing with a cw external cavity quantum cascade laser based spectrometer in the $6-11 \mu \mathrm{m}$ range
}

\author{
Robert Heinrich, ${ }^{1,2}$ Alexandru Popescu, ${ }^{2, *}$ Rainer Strzoda, ${ }^{2}$ Andreas Hangauer, ${ }^{2}$ and Sven Höfling ${ }^{1,3, \dagger}$ \\ ${ }^{1}$ Technical Physics, University of Würzburg, Am Hubland, 97074 Würzburg, Germany \\ ${ }^{2}$ Corporate Technology, Siemens AG, Otto-Hahn-Ring 6, 81739 Munich, Germany \\ ${ }^{3}$ SUPA, School of Physics and Astronomy, University of St Andrews, St Andrews KY 16 9SS, UK
}

(Dated: February 4, 2019)

\begin{abstract}
We report multi-species spectroscopy of hydrocarbons with a continuous wave external-cavity quantum cascade laser (EC-QCL) based spectrometer providing tunability from 6-11 $\mu \mathrm{m}$ to measure direct absorption spectra of the first 7 alkanes and their mixtures. The gas spectra where acquired in the range from $1440-1480 \mathrm{~cm}^{-1}$ at reduced pressure of $50 \mathrm{mbar}$ and at a temperature of $323 \mathrm{~K}$. By linearization of the measured wavelengths with a custom-made highly temperature stable air spaced etalon high spectral accuracy of $\pm 0.001 \mathrm{~cm}^{-1}$ is achieved for the whole spectral range. The simultaneous high resolution of $0.001 \mathrm{~cm}^{-1}$ yields spectra of unprecedented richness of detail for the heavier alkanes $\left(\mathrm{C}_{3}-\mathrm{C}_{5}\right)$ and allows the discrimination of narrow spectral features for the lighter ones $\left(\mathrm{C}_{1}-\mathrm{C}_{2}\right)$. Thereby, the measured spectra reveal the influences of collisional broadening effects among the measured species. Quantitative spectroscopic multi-species gas sensing relies on the comprehension of the extent of spectral broadening. Studying the spectral broadening in combination with highly accurate reference spectra is mandatory for highly sensitive and specific multi-species gas analyzers. The quantitative results that can be obtained with our approach are presented for an exemplary calibrated mixture of all 7 components and reveals an absolute accuracy below $0.5 \mathrm{vol} \%$ for the determination of the mole fraction of each gas.
\end{abstract}

\section{Introduction}

Quantitative spectroscopy of individual hydrocarbons as well as of their mixtures is of great interest for a multitude of applications. These include the detection of atmospheric composition change [1,2], pipeline gas leak detection [3], exhaled breath analysis $[4,5]$ and especially the quantification of alkane mixtures in process streams [6-8] of the petrochemical industry. In the latter example gas chromatographs are the most widely employed technology for process analysis by decomposing the gas stream while measuring the concentration of each constituent [9]. But in contrast to spectroscopic systems GCs are dependent on sophisticated and cost-intensive calibration systems making research on other approaches for mulit-species gas analysis worthwhile.

The spectroscopic quantification of gas mixtures frequently requires the coverage of a broad spectral range to include the respective absorption lines of the different species and at, ideally, a high spectral resolution to allow for discrimination of partially overlapping absorption lines. In the past different approaches were used for the spectroscopic quantification of hydrocarbons. This includes Vis-NIR-spectroscopy [10], Fourier transform infrared spectroscopy (FTIR) [6] and the application of tunable filtometers [11] in the mid-infrared. The strongest fundamental absorption bands in the infrared for the alkanes are found at $3.3 \mu \mathrm{m}$ wavelength attributed to $\mathrm{CH}$ stretching vibrations and around $6.8 \mu \mathrm{m}$ wavelength due to bending vibrational modes of the $\mathrm{CH}$ bonds [12].

For laser based multi-species gas sensing a setup with a pulsed vertical external cavity surface emitting laser
(VECSEL) in the $3 \mu \mathrm{m}$ range was demonstrated to detect mixtures of the first 4 alkanes down to the ppm level with a resolution of $1.3 \mathrm{~cm}^{-1}$ [13]. The development of Interband Cascade Laser (ICLs) [14] based multimode absorption spectroscopy (MUMAS) $[15,16]$ revealed an elegant method for the simultaneous detection of methane, acetylene and formaldehyde in a gas mixture with relative detection uncertainties of $10 \%$. The method assumes stable laser operation and is limited when to many absorption features overlap. Trace gas level detection of formaldehyde in a mixture with methanol and formic acid in air has been realized with a single distributed feedback ICL [17] and showed good agreement with the Pacific Northwest National Laboratory (PNNL) database [18].

Quantum Cascade Lasers (QCLs) [19] were already multiply demonstrated for gas detection in the MIR fingerprint region as they can be tailored to emit at any wavelength from $3 \mu \mathrm{m}$ right up the $\mathrm{THz}$ region [20], with the exception of the Reststrahlenband of the respective semiconductor [21]. Especially, external-cavity quantum cascade lasers (EC-QCLs) take advantage of the full gain spectrum of QCLs to enable a widely tunable spectrally single mode laser due to the application of a wavelength selective grating. Different systems with EC-QCL based setups were published that accomplished sensitive and high resolution measurements of single absorption lines allowing trace gas detection in the ppb range and the discrimination of isotopes. For the measurements either long path absorption cells [22] or cavity enhanced techniques [23-25] were applied.

EC-QCLs also have the advantage that with elaborate optomechanics fast sweeps over the entire gain profile in 
a sub-second timescale are feasible. This allows for realtime trace gas sensing of several components with broad absorption features as demonstrated by Phillips et al. for four different fluorocarbons [26]. But as the external cavity (EC) length is fixed for fast sweep operation the spectral resolution is dependent on the EC modes and thus more appropriate for molecules with broad absorption features. Thus, direct comparisons of performance values as achievable accuracy and integration time is limited.

While these setups were optimized for highly sensitive trace gas detection [27], high spectral resolution can be realized by techniques based on referencing of QCLs with NIR frequency combs. These measurements allowed absolute spectroscopy of multiple lines of $\mathrm{CO}_{2}[28,29]$ and $\mathrm{N}_{2} \mathrm{O}$ [30] for studying line profile models and the highly accurate determination of line center positions. For this technology the employed setups are very complex since they require the QCL emission for absorption measurements of the gas species and the NIR frequency comb source for spectral referencing.

The sensing of multi-species gas mixtures requires to comply with several of these parameters ranging from a broad wavelength coverage to a high wavelength resolution and a high sensitivity. The measurements that are presented in the following sections accomplish: 1) a broad spectral coverage of the bending vibrational modes of the targeted alkanes $\left(\mathrm{C}_{1}-\mathrm{C}_{5}\right)$ around $6.8 \mu \mathrm{m}$ for simultaneous detection of characteristic absorption features of the multiple components, 2) to reach a measurement sensitivity high enough to allow for measuring over a wide dynamic range from permille to $100 \%$ concentration of each gas, 3) to ensure a high spectral resolution and accuracy required to discriminate the overlapping absorption lines.

We present transmission spectra of pure alkanes as of their mixtures measured with an EC-QCL based setup tunable from 6-11 $\mu \mathrm{m}$. The spectral accuracy is increased with a custom-made highly temperature stable etalon yielding spectra with an accuracy of $\pm 0.001 \mathrm{~cm}^{-1}$. We carried out measurements of collisional broadening among the measured hydrocarbons in binary mixtures. Collisional broadening among hydrocarbons has not been studied in detail in the literature yet but is essential for an accurate quantitative sensing of high concentration multi-component mixtures. We delineate the influence of collisional broadening on the spectral lines of methane and deduce a method for quantitative sensing that works despite the mutual broadening.

\section{Experimental details and signal processing}

A detailed description of the experimental setup and the measurement procedure has been published previously [8]. Briefly summarizing, a EC-QCL system (Daylight Solutions, MIRcat-1400) tunable from 6-11 $\mu \mathrm{m}$

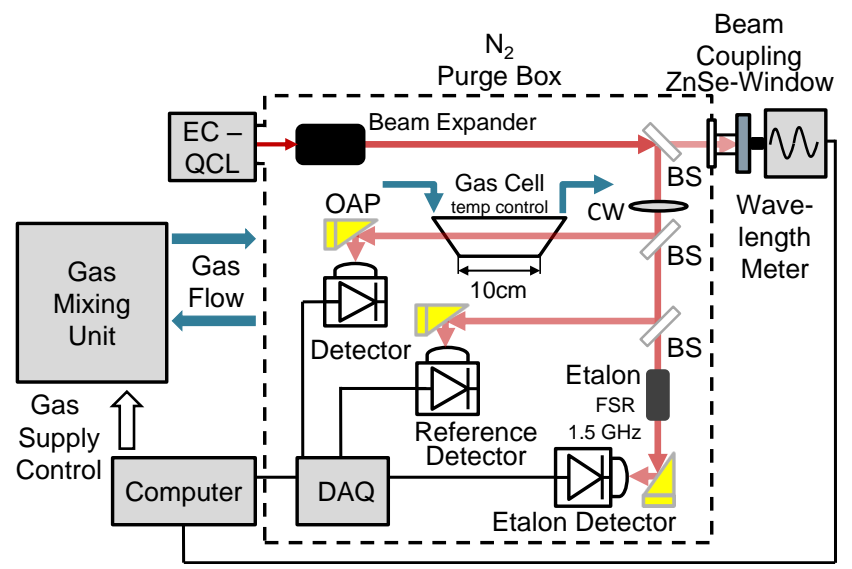

Figure 1. Schematic of the measurement system (for details cf. [8]). Component abbreviations: external cavity quantum cascade laser $(E C$ - $Q C L)$, beam splitter $(B S)$, mechanical chopper wheel $(C W)$ and off-axis parabolic mirror $(O A P)$

was employed while a high resolution Wavelength Meter (HighFinesse, WS6-200 IR-3) with 4 MHz resolution and $200 \mathrm{MHz}$ absolute accuracy served for referencing and sorting the emission by wavelength of the only quasicontinuously tunable, thus mode-hopping, laser. A regular one point calibration of the wavelength meter with a reference laser system (HighFinesse, SLR-1532) locked to an acetylene absorption line with a nominal output of $1.523 \mu \mathrm{m} \pm 0.07 \mathrm{pm}$ provides stable measurement conditions. The laser beam was split to allow coupling to the wavelength meter and to three MCT detectors (Vigo System, PVM-2TE-8). One detector acquires the transmission signal through a temperature controlled single path gas cell, the second serves as reference and the third one records the transmission signal of an etalon for relative wavelength referencing and data rejection. Due to the fixed cavity lengths in the laser system a method of stepwise temperature tuning was implemented (for details cf. Ref. [8]) This procedure could realize gapless coverage with a resolution of $0.001 \mathrm{~cm}^{-1}$ but implies long measurement times.

For the results presented in this paper a few changes of the setup have been realized: the formerly $1 \mathrm{~cm}$ short path gas cell was replaced by a $10 \mathrm{~cm}$ path temperature controlled gas cell (cf. Fig. 1). Spectral measurements are performed at constant gas flow while the gases can be heated up to $200{ }^{\circ} \mathrm{C}$. The high resolution pressure controller behind the gas cell was complemented by a high precision manometer with a pressure range of 0 to 1000 mbar, \pm 0.5 mbar accuracy and \pm 0.25 mbar longtime stability of the measured pressure point.

For referencing of the wavelength emitted by the ECQCL the wavelength meter guarantees a spectral accuracy of at least $200 \mathrm{MHz}$ over a broad range of $2-11 \mu \mathrm{m}$. In order to further improve the spectral measurement accuracy we replaced the germanium(Ge)-etalon in the 
setup from Ref. [8] by a custom-made air spaced etalon with wedged Ge windows. The one inch diameter windows are separated by a $10 \mathrm{~cm}$ hollow core Zerodur cylinder yielding a free spectral range (FSR) of $0.05 \mathrm{~cm}^{-1}$ or $1.5 \mathrm{GHz}$. Due to the extremely low thermal expansion coefficient $\alpha$ of the Zerodur of $0 \pm 0.05 \mathrm{ppm} / \mathrm{K}$ for a temperature range from $0^{\circ}$ to $50^{\circ} \mathrm{C}$ we obtain a quasistable wavelength reference. An analysis of the airy-peak spacing of the etalon transmission that is assigned to the wavelength axis of the wavemeter acquisition reveals deviations from a constant FSR. For the correction of the distorted wavelength axis we calculate the analytic signal of the recorded transmission with the Hilbert transform and subsequently apply a multiplication with a synthetic complex Airy function with constant FSR.

The analytic signal is a complex-valued function with no negative frequency components. Therefore, in the spectrum of a Fourier transform the negative frequencies can be discarded with no loss of information. We calculate the Fourier transform of the product of the analytic signal of our measured etalon transmission with the synthetic complex etalon transmission. In the FFT spectrum we only keep the components around the main frequency that corresponds to the modulation frequency of the etalon. After this frequency filter an inverse FFT is calculated from which the phase difference between the analytic signal and the synthetic etalon transmission can be extracted. This procedure is analogous to a complex demodulation of the etalon transmission signal [31].

The conversion of the phase information to the wavenumber scale of the measured spectra and subsequent correction increases the spectral precision of the measurements to $\pm 0.001 \mathrm{~cm}^{-1}$ or $\pm 30 \mathrm{MHz}$. Figure 2 illustrates the correction for a small sample set of an etalon transmission measurement. The phase shift that

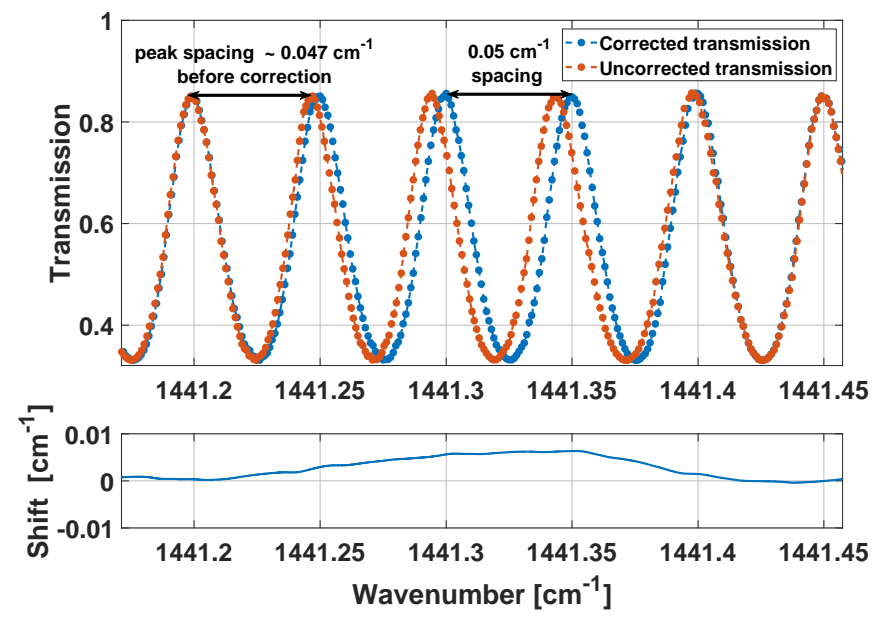

Figure 2. Exemplary phase correction of etalon transmission with uncorrected and corrected signal (upper graph) and the phase shift applied to the uncorrected signal (lower graph). is applied to the measured uncorrected transmission is shown in the lower graph and the uncorrected and corrected transmission signal are shown on the upper one.

Finally, an offset depending on the wavelength meter accuracy remains. The acquisition of methane spectra at the measured wavelength range allows to determine the offset between the corrected methane transmission spectrum and a simulated transmission spectrum by referencing to tabulated high accuracy line positions from the HITRAN database [32]. It was examined that this offset is stable in the $30 \mathrm{MHz}$ range for at least one hour and only shows slight drifts with room temperature which is monitored. Thereby, we can correct the remaining offset also for non methane spectra.

This procedure enables us to obtain spectra of an absolute accuracy of $\pm 0.001 \mathrm{~cm}^{-1}$, which is much higher then reported before in Ref. [8].

\section{Results}

In this section we emphasize the accuracy of our measurements by comparing the repeated transmission measurement of a methane multiplet with a simulated HITRAN [32] spectrum. For the targeted spectral region the database states an accuracy of the line positions of $0.14-0.55 \times 10^{-3} \mathrm{~cm}^{-1}$ and relative line intensity variations in the range from $3 \%$ to $5 \%$ for theoretical modeling of measurement data of methane [33]. Deviations may arise due to limited data for line broadening, line mixing, speed dependence and collisional narrowing which would require not-yet available "non-Voigt" line shape parameters.

The calculation of the Allan-Werle deviation [34] reveals the stability of the measurements. Afterwards, we present exemplary transmission measurements of pure methane, propane, butane and iso-butane as of their binary mixtures. These binary spectra exemplify the extent of spectral broadening for these gases. Finally, we demonstrate the capability of quantitative measurements for an exemplary mixture of all 7 alkanes. All measurements were acquired at $50 \pm 0.25$ mbar gas pressure and $323 \pm 0.3 \mathrm{~K}$ gas temperature.

\section{Spectral accuracy and Allan deviation}

As described before the wavelength meter measurements were linearized by demodulation of the etalon transmission. This correction yields an uncertainty of the measured wavenumber of $\pm 0.001 \mathrm{~cm}^{-1}$ that was confirmed by the precision of successively acquired spectra for all measured wavelength ranges.

In the following we demonstrate the high accuracy by comparison of the measured transmission of a methane multiplet with a simulated transmission in Figure 3 . The 
simulated curve was calculated with a Voigt profil taking the line positions, strengths and self-broadening parameters tabulated in the HITRAN database [32]. We averaged the acquired spectra of 40 successive measurements over an acquisition time of two hours. The residual in Figure 3 shows an obvious deviation in the range of 1-2\% transmission at the two mean peaks of the overlapping multiplet lines while the baseline and the small side-peak at $1440.967 \mathrm{~cm}^{-1}$ show minor residual values. The residual is in the range of the previously outlined uncertainties of the methane line intensities in the HITRAN database. Concurrently, the low residual beside the main peaks and the coinciding peak position of measured and simulated transmission confirm the high accuracy and repeatability of the measurements.

In order to determine the limits of averaging we calculated the Allan-Werle deviation for a longtime series of consecutive methane transmission measurements. The first spectrum was taken as reference. We assumed that the reference spectrum $S_{\text {ref }}$ equals to a consecutive spectrum $S_{n}$ but for an exponential parameter $a$ that corresponds to deviations in the spectral form and an exponential parameter $\mathrm{b}$ that corresponds to an offset of the baseline of the spectrum as given in equation 1 .

$$
S_{n}(\nu)=I_{n}(\nu) \mathrm{e}^{-A_{n}(\nu)}=\left(S_{r e f}(\nu)\right)^{a} e^{b}
$$

$S_{n}$ corresponds to the Lambert-Beer law with the incident intensity $I_{n}$ and an absorbance of the transmitted gas $A_{n}$. This equation can be linearized by applying a natural logarithm on both sides of the equation:

$$
\ln \left(S_{n}(\nu)\right)=a \cdot \ln \left(S_{r e f}(\nu)\right)+b
$$

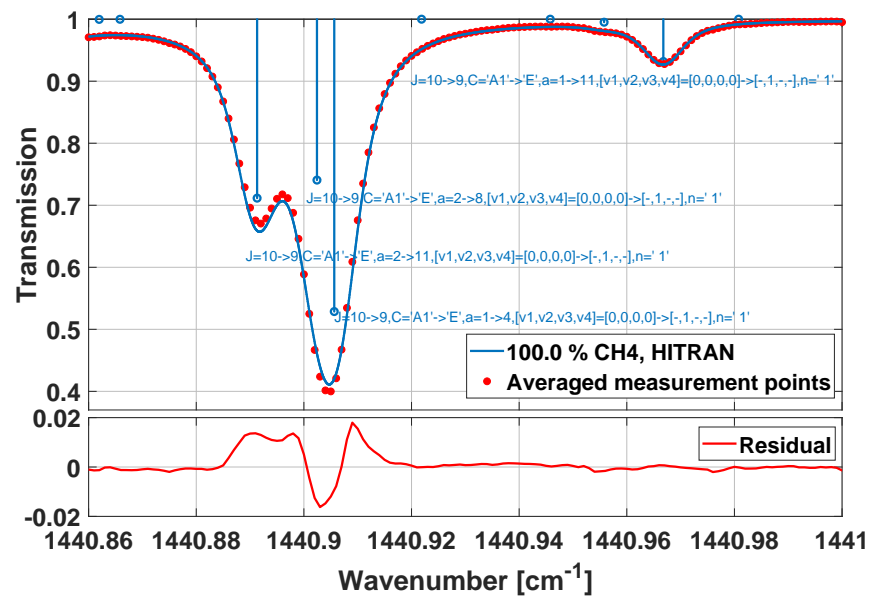

Figure 3. Comparison of the average of 40 successively acquired methane spectra and the HITRAN simulated transmission for this multiplet of the $\nu_{2}$ band (upper graph). The notation for the ro-vibrational transitions is stated according to the HITRAN database [32]. The residual is in the range of the database uncertainties for methane (lower graph).

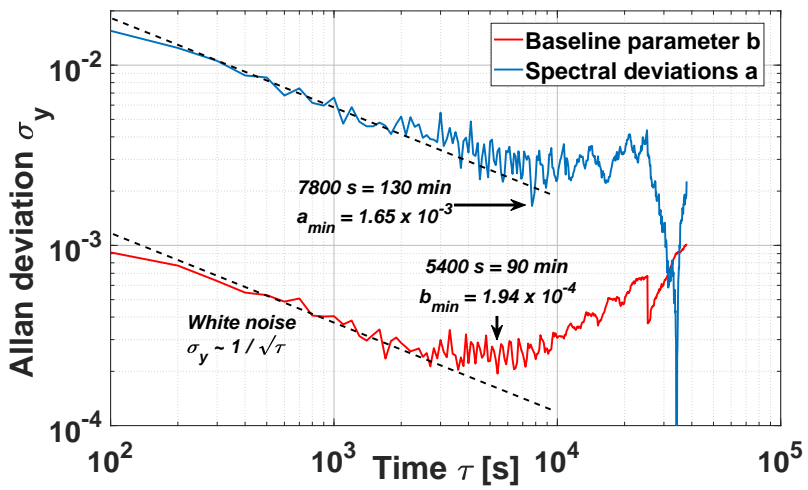

Figure 4. Allan-Werle deviation plots of the parameters $a$ and $b$ (cf. Eq. 2) of methane transmission measurements. Parameter $b$ corresponds to a noise-equivalent transmission of the spectral baseline. The minimum $b_{\min }$ of $1.94 \times 10^{-4}$ is reached after $5400 \mathrm{~s}$ measurement time while an average plateau of $2.5 \times 10^{-4}$ from 3000 to $7000 \mathrm{~s}$ indicates the reasonable measurement time for averaging. The subsequent drift is most likely due to temperature changes in the setup environment.

In this way $a$ and $b$ become linear parameters in the equation system for all measured spectra $S_{n}$ that can be solved. Parameter $b$ equals to a baseline drift of the consecutively measured spectra. The temporal evolution of the Allan-Werle deviations of $a$ and $b$ are depicted in Figure 4 and compared to the theoretical trend of purely white noise averaging. The deviation of the baseline drift parameter $b$ yields a minimum noise-equivalent transmission of $1.94 \times 10^{-4}$ after $5400 \mathrm{~s}$ total acquisition time. The deviation is given by:

$$
\sigma_{y}(\tau)=\sqrt{\sigma_{y}^{2}(\tau)}=\sqrt{\frac{1}{2 m} \sum_{n=1}^{m}\left(\bar{y}_{n+1}-\bar{y}_{n}\right)^{2}}
$$

where $\tau$ is the averaging time and $\bar{y}_{n}$ the average of the $n$th subensemble of the respective peak normalized spectral parameters $y=\{a, b\}$ of the spectra measured during the time interval $[n \tau,(n+1) \tau]$ and $m$ the number of subensembles at averaging time $\tau$. The Allan-Werle plot for parameter $a$ shows a longer continuing white noise behavior. Parameter $a$ displays the spectral shape deviation over consecutive spectra which mainly originates from the wavenumber uncertainty of $\pm 0.001 \mathrm{~cm}^{-1}$. The constantly decreasing deviation over long averaging times illustrates the benefit of the wavelength correction and confirms the repeatability of the measurements.

This confirms that despite the long acquisition time averaging of the spectra is reasonable as our setup provides stable measurement conditions in the scale of one to two hours. Hence, all spectra that we present in the following subsection were averaged for ten successive measurements which corresponds to approx. one hour acquisition time. 
Spectral broadening

Spectral databases (e.g. HITRAN [32], PNNL [18], GEISA [35], HITEMP [36]) include a large amount of line parameters and measured cross-section for a multitude of species over broad spectral ranges. These spectral compilations are continuously extended and improved, with respect to data coverage as well as to data accuracy. But as the comparison of the measured methane spectra with the simulated HITRAN profile emphasizes (cf. Fig. 3), beside that accurate line profiles are ongoing subject of current research, spectral parameters and cross-sections also may have limited accuracy. For example recent investigation of the ethane spectrum in the $7 \mu \mathrm{m}$ region [37] stated uncertainties of the computed line intensities in the range of $10 \%-20 \%$. Despite that the line positions for methane have been determined to a very high accuracy, line strength, line shift and broadening parameters especially in gas mixtures are only partially studied, yet. For heavier hydrocarbons $\left(\mathrm{C}_{3}-\mathrm{C}_{5}\right)$ the PNNL database includes cross-section data acquired at $1 \mathrm{~atm}$. Consequently, the tabulated spectra are pressure broadened while specific line positions and parameters are unknown.

In this research we particularly target two deficiencies of the available databases: 1) the collisional broadening of the targeted hydrocarbons among each other, 2) the unknown transmission profile of the heavier hydrocarbons $\left(\mathrm{C}_{3}-\mathrm{C}_{5}\right)$ at low pressures due to limited knowledge in their line parameters and absorption cross-sections.

In order to investigate the mutual broadening of the absorption lines it is essential to start with binary gas mixtures. Line positions, strength and broadening parameters are tabulated in HITRAN for methane and ethane which allows fitting of their line profiles to the measured spectra. In former studies methane lines of the $2 \nu_{3}$ band around $1.64 \mu \mathrm{m}$ and their broadening and shift coefficients in air and noble-gases were comprehensively analyzed [38-40]. Zéninari et al. [38] compared the measured broadening and shift coefficients of the methane lines regarding the overall pressure, atomic mass and polarizability of the perturbing species. In general line broadening and shift linearly decrease with the partial gas pressure of the perturber species while perturbing molecules with higher atomic mass or higher polarizability lead to stronger broadening and larger shifts of the methane lines. But broadening of hydrocarbons among each other was not specifically analyzed in former research.

In our studies we performed a simplified analysis of the broadening of several methane lines around $1449 \mathrm{~cm}^{-1}$ depicted in Fig. 6. Therefore, we assumed an equivalent broadening for all considered lines, thus having the same linear slope of line broadening with increasing perturber concentration for all lines. Thus, by extrapolation

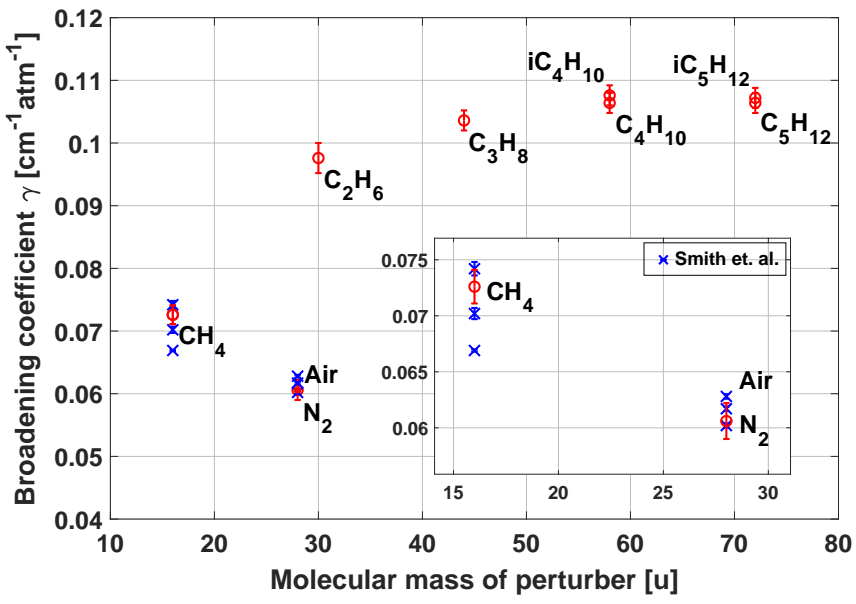

Figure 5. Methane line broadening parameter $\gamma$ for selfbroadening and different perturber gases. The broadening species is indicated by its chemical formula and the gases are sorted according to their molecular mass $\mathrm{u}$. The studied methane lines around $1449 \mathrm{~cm}^{-1}$ are part of the $\nu_{2}$ band of the methane dyad (cf. Fig. 6). For simplification we depict the mean values of the half widths of the considered lines as circles with their uncertainties. Previous results for the half width for self-broadening and broadening in air by Smith et al. [41] for three of the investigated lines are depicted as crosses. The inset shows a magnification comparing the previous results for the half widths with our measurements.

the theoretical broadening coefficient for $100 \%$ perturber gas concentration was calculated.

We could confirm the findings of Ref. [38] as the methane lines measured here also increasingly broaden for collisional partners with growing carbon atom number (see Fig.5). The transmission was measured for methane in binary mixtures with nitrogen and the stated hydrocarbons at different mixing ratios. The self-broadened line widths from the measured pure methane transmission spectrum was fitted to obtain the self-broadening coefficient for the individual lines. We compared the mean of the determined line widths for the nitrogenbroadening and self-broadening of methane with results from Smith et al. [41] (cf. Fig. 5) who did a comprehensive study of air- and self-broadening of the $\nu_{2}$ band of methane at temperatures from $225 \mathrm{~K}$ to $296 \mathrm{~K}$ and various reduced pressures. In their study they assume $1 \%$ uncertainty for their half width coefficients. For the nitrogen broadening we determined a mean halfwidth of $0.0606 \mathrm{~cm}^{-1} \mathrm{~atm}^{-1}$ and for the self-broadening $0.0726 \mathrm{~cm}^{-1} \mathrm{~atm}^{-1}$ which yields a deviation of smaller than $4 \%$ compared to the air-broadened and smaller than $8 \%$ for the self-broadened individual line widths that were ascertained by Smith et al.

Due to the elevated temperature of $323 \mathrm{~K}$ in our measurement and by applying the temperature dependence coefficients from Ref. [41] on average a reduced half width of $9 \%$ in air and of $2 \%$ in methane should be expected. 


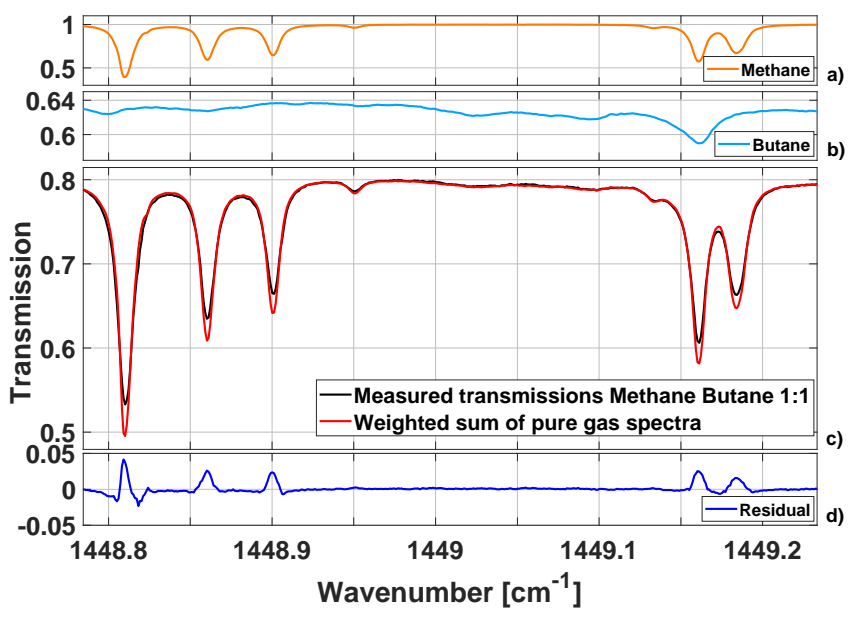

Figure 6. Methane line broadening by butane of a 1:1 mixture. Graphs a) and b) pure spectra of methane and butane, c) measured transmission of a 1:1 methane:butane mixture and weighted sum from pure spectra, d) residual between measured transmission and weighted sum of pure spectra revealing the broadening of the methane lines.

Considering that we compare the mean of the determined values we conclude a reasonable consistency of our results.

For nitrogen as perturber the line broadening reduces with increasing concentration while it increases from $\mathrm{C}_{1}$ to $\mathrm{C}_{5}$ and saturates for the heavier hydrocarbons $\left(\mathrm{C}_{4}-\mathrm{C}_{5}\right)$. Moreover, no spectral shift of the investigated lines could be measured independent of the perturber species. As former studies of different methane bands suggest spectral shifts decrease with decreasing band center wavenumber being more pronounced in the near infrared [42, 43]. For the methane dyad a crude estimation of air-broadened pressure induced shifts was given by Brown et al. [43] with $-0.002 \nu_{i} / 1300 \mathrm{~cm}^{-1} \mathrm{~atm}^{-1}$ ( $\nu_{i}$ being the line position). Therefore, even though shifts by hydrocarbon perturbers have not been studied before we expect a possible shift to be smaller than the spectral resolution of our setup as our spectra are acquired at 50 mbar.

However, in Figure 6 we demonstrate the broadening of the studied methane lines of the $\nu_{2}$ band in a 1:1 mixture with butane. The graphs $6 \mathrm{a}$ and $6 \mathrm{~b}$ show the measured transmission of the pure gases while the graph $6 \mathrm{c}$ below displays the measured transmission by the black line and by the red line the weighted sum of the transmissions from the pure gas spectra for the 1:1 mixture. The weighted sum was calculated according to the LambertBeer law (cf. Eq.4). With the concentration weighted sum of the absorbances $A_{n}$ of the measured pure reference spectra a synthetic transmission $I_{1: 1}$ of the mixture was calculated that neglects the mutual line broadening in

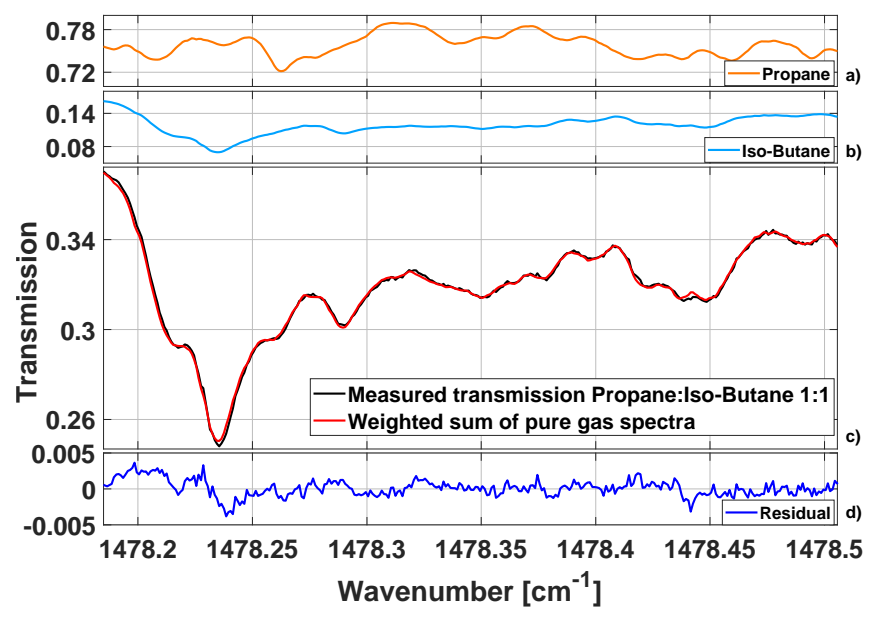

Figure 7. Spectrum of propane and iso-butane of a 1:1 mixture. Graphs a) and b) pure spectra of propane and isobutane, c) measured transmission of a 1:1 propane iso-butane mixture and weighted sum from pure spectra, d) residual between measured transmission and weighted sum of pure spectra revealing no obvious single line broadening.

the mixture:

$$
I_{1: 1}(\nu)=\mathrm{e}^{-\left(A_{1}(\nu) / 2+A_{2}(\nu) / 2\right)}
$$

The residual of measured and synthetic spectrum in graph $6 \mathrm{~d}$ obviously shows a broadening of the methane lines. The butane sample has a comparably smooth constant transmission due to a multitude of overlapping lines. A broadening effect of these lines cannot be observed from this measurement.

In comparison we present the spectra of a 1:1 propane iso-butane mixture in Figure 7. A distinct broadening is not obvious as the scale of the residual reveals deviations which are one order smaller than that for the methane butane mixture. Concurrently, individual line broadening cannot be distinguished as line position and strength for both gases are unknown while collisional effects appear to be much weaker. We assume the broadening to be less distinct since the effect is probably simultaneously spread over numerous strongly overlapping absorption lines. Thus, leading to no clear deviations in the residual of the spectra.

These results lead to the conclusion that the broadening of the lighter molecules $\left(\mathrm{C}_{1}-\mathrm{C}_{2}\right)$, which shows to be more pronounced and where the respective line positions are known, has to be considered for all perturbing partners in our set of gases $\left(\mathrm{C}_{1}-\mathrm{C}_{5}\right)$. These analysis are important for improving quantitative discrimination of light hydrocarbon absorption features in spectra of complex mixtures. But at the same time our investigations suggest that the assumption of an average broadening parameter for the investigated lines of the same absorption band already lead to reasonable results for the 
methane line-broadening. Although, a collisional broadening should be also expected for the heavier alkanes we measure much weaker influences on their spectra. Hence, in a first approach for quantitative measurements we ignore effects for $\mathrm{C}_{3}-\mathrm{C}_{5}$ and determine the concentrations in their mixtures by the respective weight for the sum of the measured pure spectra. Thus, we will discuss the quantitative determination of gas mixtures with all 7 alkanes by either ignoring spectral broadening or considering just the broadening of the methane lines in the following.

\section{Quantitative measurement results}

For a quantitative measurement of a gas mixture with all 7 alkanes we used a custom-made gravimetrically manufactured gas mixture from an external supplier. First the pure spectrum of each component was measured over a wavenumber range from $1440-1480 \mathrm{~cm}^{-1}$. The gases had a purity of $\geq 99.95 \%$. Within the considered wavenumber range three sections were selected that cover the most characteristic absorption features for each gas. This selection reduces the covered wavenumber range for the mixture analysis to approx. $10 \mathrm{~cm}^{-1}$ in total (cf. Table I).

The first analysis of the gas mixture was done by a weighted summation of the measured reference spectra according to the Lambert-Beer law and minimizing the difference between the measured spectrum $I_{\text {mix }}\left(\nu_{k}\right)$ and weighted sum of the pure reference spectra $I_{i}\left(\nu_{k}\right)$ with a least square cost function $\chi^{2}$ :

$$
\chi^{2}=\sum_{k}\left[I_{\operatorname{mix}}\left(\nu_{k}\right)-\exp \sum_{i} c_{i} \ln I_{i}\left(\nu_{k}\right)\right]^{2}
$$

The weight $c_{i}$ corresponds to the concentration of each component. A penalty term was added that coerced the sum of the concentrations to be $100 \%$ which is a valid first approach for a calibrated mixture with known concentrations $\left(\sum_{i} c_{i}=100 \%\right)$.

In Table I the determined mole fractions for this evaluation method are given. In the first line the mole fractions of the components in the gravimetrically manufactured mixture are stated with their respective uncertainties. In the lines below the results of the evaluation are given first for the entire measurement range covering three distinct parts, which sum up to $10 \mathrm{~cm}^{-1}$ in total and then for the individual sections R1-R3. The comparison of these results emphasizes the necessity of covering characteristic spectral features of all gases in order to get the best results.

We achieve a deviation $\leq 0.3 \%$ absolute from the calibrated value when all three sections are covered. By just measuring the first section $\mathrm{R} 1$ in the range from
$1448-1450 \mathrm{~cm}^{-1}$ one can obtain good results for the $\mathrm{C}_{1}-\mathrm{C}_{3}$ alkanes since they have characteristic absorption lines in this range. On the contrary, the transmission spectra of $\mathrm{C}_{4}-\mathrm{C}_{5}$ do not have characteristic features in this range but form a smooth background. As a consequence the least square algorithm fails to determine the concentration of these components by simply scaling their pure transmission spectra and yields strong deviations and large errors for the determined mole fractions. In a similar way one can interpret the results for the section R2 covering $1462-1468 \mathrm{~cm}^{-1}$. First, three times more information for the spectra can be evaluated and second all gases have characteristic features in this range. Consequently, the determined concentrations are much closer to the calibrated values but still less exact than the results when all regions are evaluated. Wavenumbers 1477 - 1479 emphasize again that for $\mathrm{C}_{5}$ the spectra are very similar and thereby difficult to discriminate while $\mathrm{C}_{1}$ has no strong absorption lines in this range which is why the relative error is pretty large. Last but not least, is becomes evident that ethane and propane are determined with the smallest deviations for all three sections which is due to there strong spectral feature over the entire wavenumber range from 1440 to $1480 \mathrm{~cm}^{-1}$.

In order to illustrate the remaining error between measured and weighted sum of the pure reference spectra a closeup of the spectrum is depicted in Figure 8. In the upper graph a) the measured transmission of the gas mixture (blue) is plotted together with the scaled transmission form the pure spectra (red). The graph b) below shows that the residual of both spectra is predominantly $\leq 5 \times 10^{-3}$.

These findings demonstrate the high accuracy that can be obtained for our spectra. Due to the high resolution of the measurements a simple weighted summation of the reference spectra already yields results with

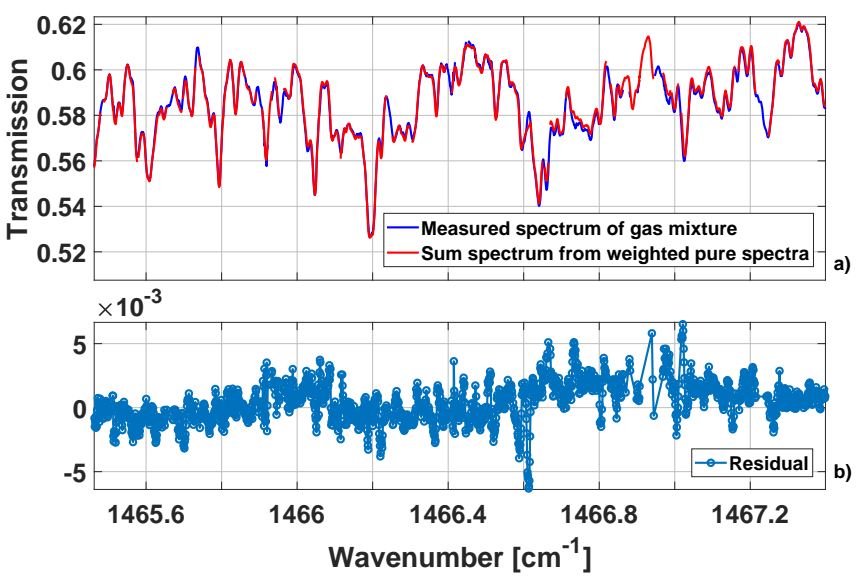

Figure 8. a): Measured transmission of calibrated gas mixture and scaled transmission from pure gas reference spectra in exemplary range of the analyzed $10 \mathrm{~cm}^{-1}$. b): Residual of measured and weighted spectrum. 


\begin{tabular}{|c|c|c|c|c|c|c|c|}
\hline Gas species in mixture & Methane & Ethane & Propane & Butane & Iso-Butane & Pentane & Iso-Pentane \\
\hline $\begin{array}{l}\text { Gravimetric mole fraction } \\
{[\mathrm{mol} / \mathrm{mol}-\%]}\end{array}$ & $4.1 \pm 0.1$ & $4.2 \pm 0.1$ & $63.7 \pm 0.2$ & $10.1 \pm 0.1$ & $9.8 \pm 0.1$ & $4.1 \pm 0.1$ & $4.0 \pm 0.1$ \\
\hline $\begin{array}{l}\text { Wavenumber range } \\
{\left[\mathrm{cm}^{-1}\right]}\end{array}$ & \multicolumn{7}{|c|}{ Determined mole fraction $[\mathrm{mol} / \mathrm{mol}-\%]$} \\
\hline $1448-1479^{*}$ & $4.1 \pm 0.1$ & $3.9 \pm 0.0$ & $63.9 \pm 0.1$ & $10.2 \pm 0.1$ & $9.8 \pm 0.0$ & $4.0 \pm 0.1$ & $4.1 \pm 0.1$ \\
\hline R1: $1448-1450$ & $3.7 \pm 0.2$ & $4.0 \pm 0.1$ & $63.6 \pm 0.3$ & $8.8 \pm 1.2$ & $7.6 \pm 2.3$ & $10.4 \pm 3.5$ & $1.8 \pm 2.3$ \\
\hline R2: $1462-1468$ & $4.2 \pm 0.3$ & $3.8 \pm 0.1$ & $63.8 \pm 0.1$ & $9.6 \pm 0.1$ & $10.9 \pm 0.2$ & $3.5 \pm 0.2$ & $4.2 \pm 0.2$ \\
\hline R3: $1477-1479$ & $2.0 \pm 2.6$ & $4.3 \pm 0.1$ & $64.0 \pm 0.5$ & $12.0 \pm 1.3$ & $9.5 \pm 0.2$ & $4.8 \pm 1.9$ & $3.4 \pm 0.3$ \\
\hline
\end{tabular}

*evaluates simultaneously all three sections R1-R3, summed up to $10 \mathrm{~cm}^{-1}$ in total

Table I. Results of the transmission spectrum of a gravimetrically manufactured gas mixture (gravimetric mole fractions stated on top). The respective mole fractions and their errors were determined for different wavelength ranges (R1-R3) by a weighted sum of the measured pure reference spectra of the gas components (cf. Eq. 5). An error $<0.1$ is indicated as \pm 0.0 . A penalty term in the evaluation guaranteed a value of $100 \%$ for the sum of all mole fractions.

minor deviations. But as we indicated in the section before a broadening of the lighter hydrocarbons has to be expected which should lead to faulty results for their concentration values. This broadening was ignored in the evaluation, yet. The reason for no evident deviation, especially for methane, was the penalty term that forced the sum of all concentrations to be $100 \%$. Such a boundary condition might be reasonable for a calibrated mixture where contamination with other gases can be excluded but if unknown mixtures are evaluated a penalty term can cause systematic errors in the evaluation. In addition, one obtains a distortion of the determined concentrations as for example methane contributes with the smallest number of lines to the entire spectrum and therefore induces the least error when its concentration slightly deviates. In order to verify of these arguments, we evaluated the spectra again without a penalty term. The respective results are given in Table II.

The sum of all concentrations equals to $98.5 \%$. Overall, the deviations for $\mathrm{C}_{2}-\mathrm{C}_{5}$ are in the same range as before or slightly increased as for propane. The most obvious deviation can be ascertained for methane with a value of $0.5 \%$ below the calibrated mole fraction. This result should be expected as a broadening of the methane lines in the mixtures occurs and consequently a smaller peak absorbance has to lead to a smaller concentration value when the self-broadened reference spectrum is taken for the evaluation. With the intention to keep the simple evaluation method, it is advantageous to introduce a simplified adaption method to take the methane broadening into account and thus improve the determination of the methane mole fraction in the mixture.

We estimate a mole fraction weighted broadened mean half-linewidth $v_{\text {mean }}$ from the values of Fig. 5 by summation of the respective broadening coefficients $\gamma_{i}$ multiplied with the mole fraction $\chi_{i}$ of each gas species and the gas pressure $p$ :

$$
v_{\text {mean }}=p \sum_{i} \chi_{i} \gamma_{i}
$$

Division of this value with the pressure $p$ of the mixture yields a mole fraction weighted mean broadening coefficient $\gamma_{\text {mean }}$ for the methane lines in the mixture:

$$
\gamma_{\text {mean }}=\frac{v_{\text {mean }}}{p} \Rightarrow \frac{\gamma_{\text {mean }}}{\gamma_{C_{1}}} \cong 1.41
$$

By taking the ratio of this value to the self-broadening coefficient of methane $\gamma_{C_{1}}$ we determined that a broadening of $141 \%$ of the self-broadened lines should be expected. The methane line positions and line parameters are tabulated in HITRAN and since we could reveal a good accordance between our measurements and a simulated HITRAN spectrum (cf. Fig. 3), we repeated the evaluation of the gas mixture transmission with a simulated methane reference spectrum whose linewidths were broadened to $141 \%$ of the self-broadened values. Thereby, the determination of the gas concentrations of the mixture stays unchanged but for methane. With the synthetic broadened spectrum a methane mole fraction of $4 \%$ was determined which is within the range of the calibrated mole fraction value $(4.1 \pm 0.1 \%)$. A weaker broadening of the simulated spectrum yields smaller values and stronger broadening leads to higher results for the methane concentration.

As next evaluation step the broadening of the ethane should be considered. In contrast to the methane adaption it can be expected that a correction of the ethane spectra will affect all concentration values as its absorption lines are spread over the whole range that we measure. But our measurements reveal an ethane broadening in binary mixtures and consequently the evaluation procedure has to consider broadening of the ethane lines when higher accuracies should be achieved. The spectrum analysis should also be extended regarding the de- 


\begin{tabular}{|c|c|c|c|c|c|c|c|}
\hline Gas species in mixture & Methane & Ethane & Propane & Butane & Iso-Butane & Pentane & Iso-Pentane \\
\hline $\begin{array}{l}\text { Gravimetric mole fraction } \\
{[\mathrm{mol} / \mathrm{mol}-\%]}\end{array}$ & $4.1 \pm 0.1$ & $4.2 \pm 0.1$ & $63.7 \pm 0.2$ & $10.1 \pm 0.1$ & $9.8 \pm 0.1$ & $4.1 \pm 0.1$ & $4.0 \pm 0.1$ \\
\hline $\begin{array}{l}\text { Measurement evaluation } \\
\text { method }\end{array}$ & \multicolumn{7}{|c|}{ Determined mole fraction $[\mathrm{mol} / \mathrm{mol}-\%]$} \\
\hline $\begin{array}{c}\text { Methane reference spectrum } \\
\text { measured }\end{array}$ & $3.6 \pm 0.2$ & $3.9 \pm 0.0$ & $63.3 \pm 0.1$ & $10.1 \pm 0.1$ & $9.5 \pm 0.0$ & $3.9 \pm 0.1$ & $4.2 \pm 0.1$ \\
\hline $\begin{array}{l}\text { Methane reference spectrum } \\
\text { simulated with line broadening }\end{array}$ & $4.0 \pm 0.2$ & $3.9 \pm 0.0$ & $63.3 \pm 0.1$ & $10.1 \pm 0.1$ & $9.5 \pm 0.0$ & $3.9 \pm 0.1$ & $4.2 \pm 0.1$ \\
\hline
\end{tabular}

Table II. Results for the gas mixture without a penalty term in the evaluation. The results for a weighted sum with an self-broadened reference spectrum of methane and with a simulated methane spectrum, that considers the line broadening by the concentration of perturber gases in the mixture, are stated. The methane mole fractions (bold) emphasize that smaller deviations can be achieved when the line broadening is considered.

termination of the broadening coefficient in the gas mixture. Although, for the evaluation here the broadening of the methane lines was fixed, in future measurements of mixtures with unknown mole fractions the broadening coefficient could be a fitting parameter. The investigation of such a method is prospect of future research and requires measurements of further gas mixtures with varying mixing ratios.

However, to our knowledge no laser-based measurement of a seven components alkane gas mixture has been reported with a comparable accuracy, yet. The chosen concentrations for the calibrated mixture were based on concentration values that occur in propane producing process streams in refineries. We expect that the measurement procedure guarantees to determine the concentrations of all components for a dynamic range from permille to $100 \%$ in mixtures with the here demonstrated accuracies. The detection limit of the pure component can be estimated from the noise level of the Allan-Werle plot and the maximum absorbance within the considered wavenumber range. However, for the weighted sum approach in mixtures the individual uncertainties of the reference spectra add together which especially hampers low concentration determination of spectra with smooth spectral shape as for the pentanes. For trace gas applications either another laser source with lower noise level or a long path cell would be required.

\section{Conclusion}

The quantitative determination of a 7 component alkane gas mixture with absolute deviations below 0.5 vol\% with a lab based setup and direct absorption spectroscopy with a broadly tunable EC-QCL was demonstrated. In the course of this research we could obtain pure gas spectra with a spectral resolution of $0.001 \mathrm{~cm}^{-1}$ and an accuracy of $\pm 0.001 \mathrm{~cm}^{-1}$ in the wavenumber range from $1440-1480 \mathrm{~cm}^{-1}$. We want to emphasize that especially for the alkanes $\mathrm{C}_{3}-\mathrm{C}_{5}$ no comparable resolution has been documented in this wavelength range, yet. Due to the low pressure of 50 mbar in our measurements we can take advantage of this resolution for the quantitative analysis in mixtures as much more structure of the spectra can be resolved. This spectral quality allowed us to compare a methane multiplet with a Voigt-profile simulated spectrum taking the line parameters from the HITRAN database. In addition we could demonstrate long time stability of the measurements with an Allan-Werle deviation plot revealing a minimum detectable absorption in the range of $10^{-4}$ for long time averaging. We studied the broadening of selected methane lines in mixtures with nitrogen and the other 6 hydrocarbons. With a simplified model collisional broadening effects have been investigated. To our knowledge no comparable studies of methane broadening with other alkanes as perturbing species has been reported in this wavelength range. Despite the rough estimation of these broadening coefficients we could demonstrate that by simulating a broadened methane spectrum with the determined values reduces the deviation for quantitative measurements of methane in a complex mixture of all 7 alkanes. The extension of this method for the broadening of ethane is prospect of future research. Thereby, we expect to even further decrease the deviations for the quantitative measurement of these gas mixtures. The current results indicate that a broadening of the C3-C5 hydrocarbons spectra is less pronounced and thus has fewer influence on the evaluation of gas mixture spectra.

Further improvements are conceivable by reducing the noise level of the measurements or simply scanning over a larger wavenumber range, as we could demonstrate that more spectral information consequently lead to higher accuracies for the quantitative determination of the gas mole fractions. The main drawback of this method with the used setup is the long measurement times that involve 
several hours to cover the considered spectral regions.

Thus, our results demonstrate the general feasibility of high accuracy quantitative measurements of complex gas mixtures based on direct absorption spectroscopy. For the realization of a demonstrator that could measure the same spectra within seconds other broadly tunable laser sources are required. Recent publications demonstrated the successful test of quantum cascade laser arrays $[44,45]$ in spectroscopic measurements. These results revealed the capability of sensing several gases but at other wavelengths than we targeted for our measurements. The sensing of the here studied gases with a QCL array would allow faster measurements and thus possibly even higher accuracies. Also wavelength modulation spectroscopy techniques [46, 47] could be used to improve the capability of the measurements for the detection of trace gas concentrations. This would significantly promote the development of new fast, robust and accurate multi-component gas analyzers as they are required in several industries.

The project has received funding from the European Unions Horizon 2020 research and innovation program under Grant Agreement No. 636930 (iCspec).

popescu.alexandru@siemens.com

sh222@st-andrews.ac.uk

[1] A. Pozzer, J. Pollmann, D. Taraborrelli, P. Jöckel, D. Helmig, P. Tans, J. Hueber, and J. Lelieveld, Atmospheric Chemistry and Physics 10, 4403 (2010).

[2] P. Hausmann, R. Sussmann, and D. Smale, Atmospheric Chemistry and Physics 16, 3227 (2016).

[3] J. R. Roscioli, S. Herndon, D. D. Nelson, and T. Yacovitch, Methane, Ethane, and Propane Sensor for Real-time Leak Detection and Diagnostics, Tech. Rep. (Aerodyne Research, Inc., Billerica, MA (United States), 2017).

[4] C. Wang and P. Sahay, Sensors 9, 8230 (2009).

[5] T. Stacewicz, Z. Bielecki, J. Wojtas, P. Magryta, J. Mikolajczyk, and D. Szabra, Opto-Electronics Review 24, 82 (2016).

[6] X. Tang, Y. Li, L. Zhu, A. Zhao, and J. Liu, Chemometrics and Intelligent Laboratory Systems 146, 371 (2015).

[7] X. Han, Z.-x. Huang, X.-d. Chen, Q.-f. Li, K.-x. Xu, and D. Chen, Fuel 207, 146 (2017).

[8] R. Heinrich, A. Popescu, A. Hangauer, R. Strzoda, and S. Höfling, Applied Physics B 123, 223 (2017).

[9] G. Eiceman, H. J. Hill, and J. Gardea-Torresdey, Anal Chem. 70, 321R (1998).

[10] M. A. Van Agthoven, G. Fujisawa, P. Rabbito, and O. C. Mullins, Applied Spectroscopy 56, 593 (2002).

[11] C. Gasser, A. Genner, H. Moser, J. Ofner, and B. Lendl, Sensors and Actuators B: Chemical 242, 9 (2017).

[12] G. Herzberg, Infrared and Raman spectra of polyatomic molecules (D. Van Nostrand Company.; New York, 1945).

[13] J. M. Rey, M. Fill, F. Felder, and M. W. Sigrist, Applied Physics B 117, 935 (2014).

[14] I. Vurgaftman, R. Weih, M. Kamp, J. Meyer, C. Canedy, C. Kim, M. Kim, W. Bewley, C. Merritt, J. Abell, et al.,
Journal of Physics D: Applied Physics 48, 123001 (2015).

[15] J. Northern, S. OHagan, B. Fletcher, B. Gras, P. Ewart, C. Kim, M. Kim, C. Merritt, W. Bewley, C. Canedy, et al., Optics letters 40, 4186 (2015).

[16] S. OHagan, J. Northern, B. Gras, P. Ewart, C. Kim, M. Kim, C. Merritt, W. Bewley, C. Canedy, I. Vurgaftman, et al., Applied Physics B 122, 1 (2016).

[17] S. Lundqvist, P. Kluczynski, R. Weih, M. von Edlinger, L. Nähle, M. Fischer, A. Bauer, S. Höfling, and J. Koeth, Applied optics 51, 6009 (2012).

[18] T. J. Johnson, R. L. Sams, and S. W. Sharpe, in Chemical and Biological Point Sensors for Homeland Defense, Vol. 5269 (International Society for Optics and Photonics, 2004) pp. 159-168.

[19] M. Razeghi, W. Zhou, S. Slivken, Q.-Y. Lu, D. Wu, and R. McClintock, Applied optics 56, H30 (2017).

[20] M. S. Vitiello, G. Scalari, B. Williams, and P. De Natale, Optics express 23, 5167 (2015).

[21] J. Faist, F. Capasso, C. Sirtori, D. L. Sivco, and A. Y. Cho, in Semiconductors and semimetals, Vol. 66 (Elsevier, 1999) pp. 1-83.

[22] G. Hancock, J. Van Helden, R. Peverall, G. Ritchie, and R. Walker, Applied Physics Letters 94 (2009).

[23] J. H. van Helden, N. Lang, U. Macherius, H. Zimmermann, and J. Röpcke, Applied Physics Letters 104, 099901 (2014).

[24] A. De, G. D. Banik, A. Maity, M. Pal, and M. Pradhan, Optics letters 41, 1949 (2016).

[25] A. Maity, M. Pal, G. D. Banik, S. Maithani, and M. Pradhan, Laser Physics Letters 14, 115701 (2017).

[26] M. C. Phillips, M. S. Taubman, B. E. Bernacki, B. D. Cannon, R. D. Stahl, J. T. Schiffern, and T. L. Myers, Analyst 139, 2047 (2014).

[27] G. N. Rao and A. Karpf, Applied optics 50, A100 (2011).

[28] D. Gatti, A. Gambetta, A. Castrillo, G. Galzerano, P. Laporta, L. Gianfrani, and M. Marangoni, Optics express 19, 17520 (2011).

[29] A. Gambetta, D. Gatti, A. Castrillo, G. Galzerano, P. Laporta, L. Gianfrani, and M. Marangoni, Applied Physics Letters 99, 251107 (2011).

[30] K. Knabe, P. A. Williams, F. R. Giorgetta, M. B. Radunsky, C. M. Armacost, S. Crivello, and N. R. Newbury, Optics express 21, 1020 (2013).

[31] T. Niebauer, Applied optics 52, 1838 (2013).

[32] L. Rothman, I. Gordon, Y. Babikov, A. Barbe, D. C. Benner, P. Bernath, M. Birk, L. Bizzocchi, V. Boudon, L. Brown, A. Campargue, K. Chance, E. Cohen, L. Coudert, V. Devi, B. Drouin, A. Fayt, J.-M. Flaud, R. Gamache, J. Harrison, J.-M. Hartmann, C. Hill, J. Hodges, D. Jacquemart, A. Jolly, J. Lamouroux, R. L. Roy, G. Li, D. Long, O. Lyulin, C. Mackie, S. Massie, S. Mikhailenko, H. Mller, O. Naumenko, A. Nikitin, J. Orphal, V. Perevalov, A. Perrin, E. Polovtseva, C. Richard, M. Smith, E. Starikova, K. Sung, S. Tashkun, J. Tennyson, G. Toon, V. Tyuterev, and G. Wagner, Journal of Quantitative Spectroscopy and Radiative Transfer 130, 4 (2013), hITRAN2012 special issue.

[33] L. Brown, K. Sung, D. Benner, V. Devi, V. Boudon, T. Gabard, C. Wenger, A. Campargue, O. Leshchishina, S. Kassi, et al., Journal of Quantitative Spectroscopy and Radiative Transfer 130, 201 (2013).

[34] P. Werle, R. Mücke, and F. Slemr, Applied Physics B: Lasers and Optics 57, 131 (1993). 
[35] N. Jacquinet-Husson, R. Armante, N. Scott, A. Chédin, L. Crépeau, C. Boutammine, A. Bouhdaoui, C. Crevoisier, V. Capelle, C. Boonne, et al., Journal of Molecular Spectroscopy 327, 31 (2016).

[36] L. Rothman, I. Gordon, R. Barber, H. Dothe, R. Gamache, A. Goldman, V. Perevalov, S. Tashkun, and J. Tennyson, Journal of Quantitative Spectroscopy and Radiative Transfer 111, 2139 (2010).

[37] C. Di Lauro, F. Lattanzi, L. R. Brown, K. Sung, J. Vander Auwera, A. W. Mantz, and M. A. H. Smith, Planetary and space science 60, 93 (2012).

[38] V. Zeninari, B. Parvitte, D. Courtois, V. Kapitanov, and Y. N. Ponomarev, Applied Physics B: Lasers and Optics 72, 953 (2001).

[39] G. Dufour, D. Hurtmans, A. Henry, A. Valentin, and M. Lepère, Journal of Molecular Spectroscopy 221, 80 (2003).

[40] V. Kapitanov, K. Y. Osipov, A. Protasevich, and Y. N. Ponomarev, Journal of Quantitative Spectroscopy and Radiative Transfer 113, 1985 (2012).
[41] M. Smith, D. C. Benner, A. Predoi-Cross, and V. M. Devi, Journal of Quantitative Spectroscopy and Radiative Transfer 133, 217 (2014).

[42] L. Brown, J. Margolis, J. Champion, J. Hilico, J. Jouvard, M. Loete, C. Chackerian, G. Tarrago, and D. Benner, Journal of Quantitative Spectroscopy and Radiative Transfer 48, 617 (1992), special Issue Conference on Molecular Spectroscopic Databases.

[43] L. R. Brown, D. C. Benner, J.-P. Champion, V. M. Devi, L. Fejard, R. Gamache, T. Gabard, J. Hilico, B. Lavorel, M. Loete, et al., Journal of Quantitative Spectroscopy and Radiative Transfer 82, 219 (2003).

[44] A. P. Michel, J. Kapit, M. F. Witinski, and R. Blanchard, Applied Optics 56, E23 (2017).

[45] L. Bizet, R. Vallon, B. Parvitte, M. Brun, G. Maisons, M. Carras, and V. Zeninari, Applied Physics B 123, 145 (2017).

[46] G. C. Bjorklund, Optics letters 5, 15 (1980).

[47] J. A. Silver, Applied optics 31, 707 (1992). 